\title{
The abundance of SiS in circumstellar envelopes around AGB stars
}

\author{
F. L. Schöier ${ }^{1,2}$, J. Bast ${ }^{1,3}$, H. Olofsson ${ }^{1,2}$, and M. Lindqvist ${ }^{2}$ \\ 1 Stockholm Observatory, AlbaNova University Center, 10691 Stockholm, Sweden \\ e-mail: schoier@chalmers.se \\ 2 Onsala Space Observatory, 43992 Onsala, Sweden \\ ${ }^{3}$ Leiden Observatory, PO Box 9513, 2300 RA Leiden, The Netherlands
}

Received 15 May 2007 / Accepted 6 July 2007

ABSTRACT

\begin{abstract}
Aims. Given their photospheric origin and refractive nature, SiS molecules can provide major constraints on the relative roles of dust condensation and non-equilibrium processes in regulating the chemistry in circumstellar envelopes around evolved stars.

Methods. New SiS multi-transition (sub-)millimetre line observations of a sample of AGB stars with varying photospheric C/O-ratios and mass-loss rates are presented. A combination of low- and high-energy lines are important in constraining the circumstellar distribution of SiS molecules. A detailed radiative transfer modelling of the observed SiS line emission is performed, including assessment of the effect of thermal dust grains in the excitation analysis.

Results. We find that the circumstellar fractional abundance of $\mathrm{SiS}$ in these environments has a strong dependence on the photospheric $\mathrm{C} / \mathrm{O}$-ratio as expected from chemical models. The carbon stars $(\mathrm{C} / \mathrm{O}>1)$ have a mean fractional abundance of $3.1 \times 10^{-6}$, about one order of magnitude higher than that found for the M-type AGB stars $(\mathrm{C} / \mathrm{O}<1)$ where the mean value is $2.7 \times 10^{-7}$. These numbers are in reasonable agreement with photospheric LTE chemical models. SiS appears to behave similarly to $\mathrm{SiO}$ in terms of photodissociation in the outer part of the circumstellar envelope. In contrast to previous results for the related molecule SiO, there is no strong correlation of the fractional abundance with density in the CSE, as would be the case if freeze-out onto dust grains were important. However, possible time-variability of the line emission in the lower $J$ transitions and the sensitivity of the line emission to abundance gradients in the inner part of the CSE may mask a correlation with the density of the wind. There are indications that the SiS fractional abundance could be significantly higher closer to the star which, at least in the case of M-type AGB stars, would require non-equilibrium chemical processes.
\end{abstract}

Key words. stars: AGB and post-AGB - stars: circumstellar matter - stars: late-type - stars: mass-loss

\section{Introduction}

Over the past decade it has become increasingly evident that non-equilibrium chemical processes are important in regulating the chemistry in the circumstellar envelopes (CSEs) found around evolved, mass-losing, low- to intermediate mass stars located on the asymptotic giant branch (AGB). From equilibrium chemistry calculations it is expected that the molecular content of the gas and dust that composes the CSEs to a large extent is dictated by the $\mathrm{C} / \mathrm{O}$-ratio in the photosphere of the star (see reviews by Glassgold 1999 and Millar 2003 and references therein). For M-type (O-rich) AGB stars $(\mathrm{C} / \mathrm{O}<1)$ most of the carbon atoms will be tied up in $\mathrm{CO}$ leaving an oxygen dominated chemistry with very low abundances of other carbon bearing molecules such as $\mathrm{HCN}$ and $\mathrm{CS}$. For carbon stars $(\mathrm{C} / \mathrm{O}>1)$, on the other hand, very low abundances of oxygen bearing species such as $\mathrm{H}_{2} \mathrm{O}$ and $\mathrm{SiO}$ are to be expected.

However, surprisingly large amounts of oxygen-bearing molecules such as $\mathrm{H}_{2} \mathrm{O}, \mathrm{H}_{2} \mathrm{CO}, \mathrm{C}_{3} \mathrm{O}$ and $\mathrm{SiO}$ have been found in the CSE around the carbon star IRC+10216 (Keady \& Ridgway 1993; Melnick et al. 2001; Ford et al. 2004; Hasegawa et al. 2006; Agúndez \& Cernicharo 2006; Tenenbaum et al. 2006; Schöier et al. 2006a). For SiO, this is the case for a large sample of carbon stars indicating that such "anomalous" chemistries might be a common phenomenon in carbon-rich CSEs (Schöier et al. 2006b). Various suggestions to explain the observed molecular abundances have been made including non-equilibrium chemical processes, Fischer-Tropsch catalytic processes, and evaporation of cometary bodies (Bieging et al. 2000; Melnick et al. 2001; Willacy 2004; Cherchneff 2006; Agúndez \& Cernicharo 2006). Similarly, in M-type AGB stars relatively large amounts of carbon bearing molecules such as $\mathrm{HCN}$ and $\mathrm{CS}$ have been found (Olofsson et al. 1998). Recent interferometric line observations of HCN have sorted out a long standing debate as to its origin, clearly indicating that it is effectively formed in, or close to, the photosphere also in the case of M-type AGB stars (Marvel 2005).

It is not unreasonable to expect that the grain type setups in the CSEs around mass-losing red giant stars are affected by these various chemical processes. Recently, Höfner \& Andersen (2007) have suggested that non-equilibrium processes forming both carbon and silicate grains in the winds of M-type AGB stars may help these winds to accelerate to the observed values. Such detailed calculations are currently not possible in hydrodynamical simulations for dust driven winds from M-type AGB stars containing pure silicates (Woitke 2006; Höfner \& Andersen 2007).

Observations of $\mathrm{SiO}$ line emission have been shown to be a useful probe of the formation and evolution of dust grains in CSEs, as well as CSE dynamics. A major survey of $\mathrm{SiO}$ line emission from AGB stars with varying photospheric $\mathrm{C} / \mathrm{O}$-ratios and mass-loss rates was performed by González Delgado et al. (2003) and Schöier et al. (2006b). To much surprise, detailed radiative transfer modelling reveals that the circumstellar 
Table 1. Telescope data.

\begin{tabular}{ccrccc}
\hline \hline Transition & $\begin{array}{c}\text { Frequency } \\
{[\mathrm{GHz}]}\end{array}$ & $\begin{array}{r}E_{\mathrm{up}} \\
{[\mathrm{K}]}\end{array}$ & Telescope & $\eta_{\mathrm{mb}}$ & $\begin{array}{c}\theta_{\mathrm{mb}} \\
{\left[{ }^{\prime}\right]}\end{array}$ \\
\hline$J=5 \rightarrow 4$ & 90.772 & 13 & OSO & 0.60 & 42 \\
$J=6 \rightarrow 5$ & 108.924 & 18 & OSO & 0.50 & 35 \\
$J=12 \rightarrow 11$ & 217.817 & 68 & JCMT & 0.70 & 22 \\
$J=19 \rightarrow 18$ & 344.779 & 166 & APEX & 0.70 & 18 \\
& & & JCMT & 0.63 & 14 \\
$J=20 \rightarrow 19$ & 362.906 & 183 & APEX & 0.70 & 17 \\
\hline
\end{tabular}

fractional abundance of $\mathrm{SiO}$ has no apparent correlation with the C/O-ratio of the star. For the carbon stars the fractional abundance can be several orders of magnitude higher than predicted by thermal equilibrium chemistry. Interestingly, a trend of decreasing $\mathrm{SiO}$ abundance with increasing mass-loss rate of the star, interpreted as an effect of increased adsorption of $\mathrm{SiO}$ onto dust grains with increasing mass-loss rate, i.e., the density of the envelope, was found for both the M-type and carbon star samples. These claims have been further corroborated by recent, high quality, interferometric $\mathrm{SiO}$ observations of two M-type AGB stars (Schöier et al. 2004) and the carbon star IRC+10216 (Schöier et al. 2006a) where also high spectral resolution infrared observations of ro-vibrational transitions were used.

Thus, there are strong indications that the circumstellar $\mathrm{SiO}$ line emission carries information on the complex region, both chemically and dynamically, where the mass-loss is initiated, and where the dust formation takes place. However, these conclusions still rest on somewhat loose ground, e.g., the constraints on the $\mathrm{SiO}$ abundance distribution are poor, and the relative importance of freeze-out onto dust grains, photodissociation, and circumstellar chemistry is still uncertain. Important complementary information may be obtained by observing another Si-bearing species, e.g., SiS which has been detected in both oxygen- and carbon-rich envelopes (Lindqvist et al. 1992; Bujarrabal et al. 1994; Olofsson et al. 1998). This species differs from $\mathrm{SiO}$ in the sense that it can be readily formed though gas-phase reactions in the inner wind (Scalo \& Slavsky 1980). Furthermore, it could possibly react differently from $\mathrm{SiO}$ to shocks (Cherchneff 2006), dust condensation, and photodissociation. The $\mathrm{SiS}$ lines are expected to be optically thin, as opposed to the $\mathrm{SiO}$ lines which are generally optically thick, and thus has the potential to probe material even closer to the star.

In this paper we present new (sub-)millimetre line observations of SiS for a sample of AGB stars with varying photospheric $\mathrm{C} / \mathrm{O}$-ratios and mass-loss rates. The observations are supplemented by a detailed non-LTE radiative transfer analysis in order to obtain reliable circumstellar SiS fractional abundances. The results are then compared to $\mathrm{SiO}$ abundance estimates for the same sample of stars and to predictions from available chemical models.

\section{Observations}

Multi-transition SiS line observations (Table 1) were performed during 1995-2006 using the Onsala $20 \mathrm{~m}^{\text {telescope }}{ }^{1}$ (OSO), the

1 The Onsala $20 \mathrm{~m}$ telescope is operated by the Swedish National Facility for Radio Astronomy, Onsala Space observatory at Chalmers University of technology.
Swedish-ESO submillimetre telescope ${ }^{2}$ (SEST), the JCMT telescope $^{3}$, and in July 2006 using the APEX telescope ${ }^{4}$.

The SEST, OSO and JCMT observations were made in a dual beamswitch mode, where the source is alternately placed in the signal and the reference beam, using a beam throw of about $11^{\prime}$ (SEST and OSO) or 2' (JCMT). This method produces very flat baselines. At the APEX $12 \mathrm{~m}$ telescope the observations were carried out using a position-switching mode, with the reference position located $+3^{\prime}$ in azimuth. The raw spectra are stored in the $T_{\mathrm{A}}^{\star}$ scale and converted to main-beam brightness temperature using $T_{\mathrm{mb}}=T_{\mathrm{A}}^{*} / \eta_{\mathrm{mb}} . T_{\mathrm{A}}^{\star}$ is the antenna temperature corrected for atmospheric attenuation using the chopper-wheel method, and $\eta_{\mathrm{mb}}$ is the main-beam efficiency. Regular pointing checks were made on $\mathrm{SiO}$ masers (SEST and OSO) and strong CO sources (JCMT and APEX) and typically found to be consistent with the pointing model within $\approx 3^{\prime \prime}$.

The adopted beam efficiencies, together with the FWHM of the main-beam $\left(\theta_{\mathrm{mb}}\right)$, for all telescopes and frequencies are given in Table 1 . The uncertainty in the absolute intensity scale is estimated to be about $\pm 20 \%$. In Table 1 the energy of the upper level involved in the particular transition $\left(E_{\mathrm{up}}\right)$ is also given, ranging from $13 \mathrm{~K}$ for the $J=5$ level up to $183 \mathrm{~K}$ for the $J=20$ level, illustrating the potential of these multi-transition observations to probe a large radial range of the CSE (Sect. 4).

The data were reduced by removing a low order polynomial baseline and then binned (typically to a velocity resolution of about $2 \mathrm{~km} \mathrm{~s}^{-1}$ ) to improve the signal-to-noise ratio, using $\mathrm{XS}^{5}$.

The observed spectra are presented in Fig. 1 and velocityintegrated intensities are reported in Table 2 . The intensity scales are given in main-beam brightness temperature scale $\left(T_{\mathrm{mb}}\right)$. In addition to the new data presented here we have also used SiS line intensities $(J=5 \rightarrow 4 \& J=6 \rightarrow 5)$ reported by Bujarrabal et al. (1994), Olofsson et al. (1998) and Woods et al. (2003). In total, 11 carbon stars and 8 M-type AGB stars have been detected in SiS line emission. These stars provide our sample and are listed in Table 3.

\section{SiS excitation analysis}

\subsection{Radiative transfer model}

The CSEs are assumed to be spherically symmetric, produced by a constant mass-loss rate $(\dot{M})$, and to expand at a constant velocity $\left(v_{\mathrm{e}}\right)$. The SiS excitation analysis is performed using a detailed non-LTE radiative transfer code, based on the Monte Carlo method and is described in more detail in Schöier \& Olofsson (2001). The code has been extensively tested and benchmarked against a wide variety of molecular-line radiative transfer codes in van Zadelhoff et al. (2002).

2 The SEST was located at La Silla, Chile and operated jointly by the Swedish National Facility for Radio Astronomy and the European Southern Observatory (ESO).

3 Based on observations obtained with the James Clerk Maxwell Telescope, which is operated by the Joint Astronomy Centre in Hilo, Hawaii on behalf of the parent organisations PPARC in the UK, the National Research Council of Canada and The Netherlands Organization for Scientific Research.

4 This publication is based on data acquired with the Atacama Pathfinder Experiment (APEX). APEX is a collaboration between the Max-Planck-Institut für Radioastronomie, the European Southern Observatory, and the Onsala Space Observatory.

$5 \mathrm{XS}$ is a package developed by $\mathrm{P}$. Bergman to reduce and analyse a large number of single-dish spectra. It is publically available from ftp://yggdrasil.oso. chalmers.se 
F. L. Schöier et al.: SiS in circumstellar envelopes around AGB stars
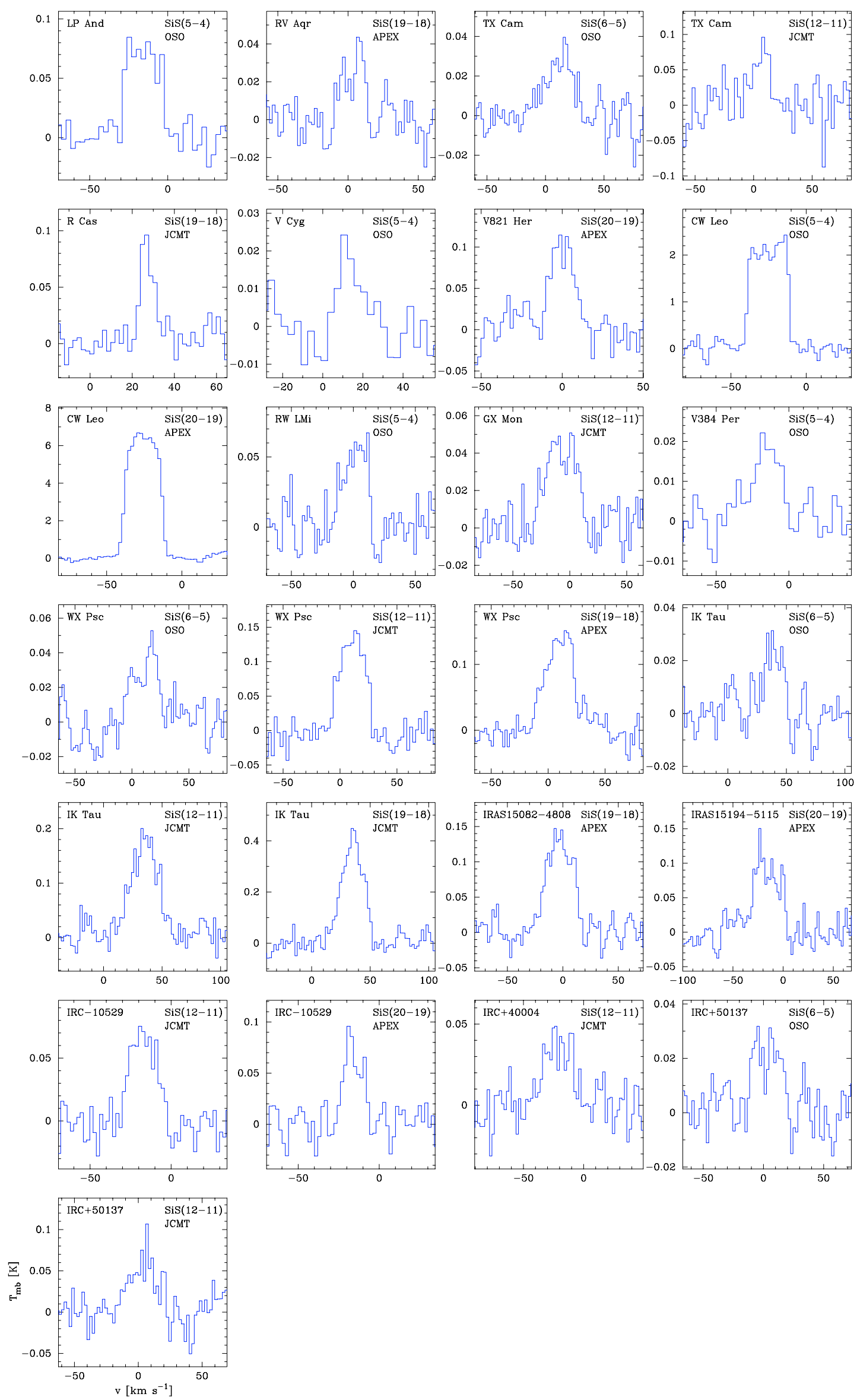

Fig. 1. New observations of $\operatorname{SiS}(v=0, J \rightarrow J-1)$ line emission. The velocity resolution is $\approx 2-3 \mathrm{~km} \mathrm{~s}^{-1}$ in all spectra. 
Table 2. Integrated $\left(I_{\mathrm{obs}}=\int T_{\mathrm{mb}} \mathrm{d} v\right)$ line intensities in $\mathrm{K} \mathrm{km} \mathrm{s}^{-1}$ for the new observations of SiS $(v=0, J \rightarrow J-1)$ line emission.

\begin{tabular}{|c|c|c|c|c|c|c|}
\hline \multirow[t]{2}{*}{ Source $^{a}$} & \multicolumn{2}{|c|}{ OSO } & \multicolumn{2}{|c|}{ JCMT } & \multicolumn{2}{|c|}{ APEX } \\
\hline & $5 \rightarrow 4$ & $6 \rightarrow 5$ & $12 \rightarrow 11$ & $19 \rightarrow 18$ & $19 \rightarrow 18$ & $20 \rightarrow 19$ \\
\hline carbon stars & & & & & & \\
\hline LP And & 1.8 & $\cdots$ & $\ldots$ & $\ldots$ & $\ldots$ & $\cdots$ \\
\hline RV Aqr & $\cdots$ & .. & ... & $\ldots$ & 0.43 & $\ldots$ \\
\hline V Cyg & $0.2:$ & $\ldots$ & $\ldots$ & $\ldots$ & $\ldots$ & $\ldots$ \\
\hline V821 Her & $\ldots$ & $\ldots$ & $\ldots$ & $\ldots$ & $\ldots$ & 1.9 \\
\hline CW Leo & 60 & $\ldots$ & $\ldots$ & $\ldots$ & $\ldots$ & 160 \\
\hline RW LMi & 1.2 & $\ldots$ & $\ldots$ & $\ldots$ & $\ldots$ & $\ldots$ \\
\hline V384 Per & 0.5 & $\cdots$ & $\ldots$ & $\ldots$ & $\ldots$ & $\ldots$ \\
\hline IRAS $15082-4808$ & $\ldots$ & $\ldots$ & $\ldots$ & $\ldots$ & 3.8 & $\ldots$ \\
\hline IRAS 15194-5115 & $\cdots$ & $\cdots$ & $\cdots$ & $\cdots$ & $\cdots$ & 3.0 \\
\hline M-type & & & & & & \\
\hline TX Cam & $\cdots$ & 0.80 & 1.5 & $\cdots$ & $\cdots$ & $\ldots$ \\
\hline R Cas & $\cdots$ & $\ldots$ & $\ldots$ & 0.8 & $\cdots$ & $\ldots$ \\
\hline GX Mon & $\ldots$ & $\ldots$ & 1.4 & $\ldots$ & $\ldots$ & $\ldots$ \\
\hline WX Psc & $\ldots$ & 1.0 & 4.0 & $\ldots$ & 4.3 & $\ldots$ \\
\hline IK Tau & $\ldots$ & 0.40 & 4.7 & 9.2 & $\ldots$ & $\ldots$ \\
\hline IRC-10529 & $\cdots$ & $\ldots$ & 1.3 & $\cdots$ & 1.0 & ... \\
\hline IRC +40004 & $\cdots$ & $\ldots$ & 1.1 & $\ldots$ & $\cdots$ & $\ldots$ \\
\hline IRC +50137 & $\ldots$ & 0.65 & 1.5 & $\ldots$ & $\ldots$ & $\ldots$ \\
\hline
\end{tabular}

${ }^{a}$ A colon (:) marks a low $\mathrm{S} / \mathrm{N}$ detection.

The circumstellar physical properties of the gas, such as the density, temperature, and kinematic structures, of the sample stars is based on radiative transfer modelling of multitransitional (sub-)millimetre $\mathrm{CO}$ line observations. The $\mathrm{CO}$ data used in the analysis have been presented in Schöier \& Olofsson (2001), Olofsson et al. (2002), González Delgado et al. (2003), and Schöier et al. (2006b). The kinetic temperature structure is obtained in a self-consistent manner from solving the energy balance equation, where the CO line cooling is directly obtained from the excitation analysis. The local line width is assumed to be described by a Gaussian and is made up of a micro-turbulent component with a Doppler width of $1.0 \mathrm{~km} \mathrm{~s}^{-1}$ ( $e$-folding radius) and a thermal component which is directly calculated from the derived kinetic temperature structure.

The excitation analysis includes radiative excitation through the first vibrationally excited $(v=1)$ state for $\mathrm{CO}$ at $4.6 \mu \mathrm{m}$ and $\mathrm{SiS}$ at $13 \mu \mathrm{m}$. Both the central star (approximated by a blackbody) and thermal dust grains distributed in the CSE can provide sufficient radiation fields which start to populate the $v=1$ state, and thereby affect the excitation in the ground vibrational state. Relevant molecular data are summarised in Schöier et al. (2005b) and are made publicly available through the Leiden Atomic and Molecular Database (LAMDA) ${ }^{6}$. The collisional rate coefficients have been extended to include more energy levels as well as extrapolated in temperature, as described in Schöier et al. (2005b). An ortho-to-para ratio of 3.0 was adopted when weighting together collisional rate coefficients for $\mathrm{CO}$ in collisions with ortho- $\mathrm{H}_{2}$ and para- $\mathrm{H}_{2}$. For $\mathrm{SiS}$ the same set of collisional rate coefficients as calculated for $\mathrm{SiO}$ has been adopted (Schöier et al. 2005b).

The majority of sources in this study are intermediate to high-mass-loss-rate objects where thermal dust emission provides the main source of infrared photons which excite the $v=1$ state. The addition of a dust component in the Monte Carlo scheme is straightforward as described in Schöier et al. (2002). The dust-temperature structure and dust-density profile are obtained from detailed radiative transfer modelling using Dusty

\footnotetext{
${ }^{6}$ http://www.strw. leidenuniv.nl/ moldata
}

(Ivezić \& Elitzur 1997). In the modelling, where the SED provides the observational constraint, the dust optical depth specified at $10 \mu \mathrm{m}, \tau_{10}$, and the dust condensation temperature, $T_{\mathrm{c}}=T_{\mathrm{d}}\left(r_{\mathrm{i}}\right)$, are the adjustable parameters in the $\chi^{2}$-analysis. The effective stellar blackbody temperature, $T_{\star}$, is usually less well constrained for these intemediate to high mass-loss-rate objects. The SED typically consists of JHKLM photometric data (Kerschbaum 1999, and Kerschbaum priv. com.), IRAS fluxes, and in some cases sub-millimetre data (Groenewegen et al. 1993). The total luminosity $\left(L_{\star}\right)$ of the source is obtained from the period-luminosity relation of Groenewegen \& Whitelock (1996) and the distance is obtained from the SED fitting. For the sources where good Hipparcos parallaxes exist, the corresponding distance has been adopted and the luminosity is then obtained from the SED modelling. Amorphous carbon dust grains with the optical constants given in Suh (2000) are adopted for the carbon stars while astronomical silicates are adopted for the M-type AGB stars. For simplicity, the dust grains are assumed to be of the same size (a radius, $a_{\mathrm{d}}$, of $0.1 \mu \mathrm{m}$ ), and the same mass density $\left(\rho_{\mathrm{s}}=2.0 \mathrm{~g} \mathrm{~cm}^{-3}\right)$. The corresponding dust opacities, $\kappa_{v}$, were then calculated from the optical constants and the individual grain properties using standard Mie theory (Bohren \& Huffman 1983).

The parameters obtained from the CO excitation analysis and the dust modelling describing the physical properties of the CSEs are reported in Table 3.

\subsection{SiS abundance distribution}

The SiS fractional abundance distribution is assumed to be described by a Gaussian

$f(r)=f_{0} \exp \left(-\left(\frac{r}{r_{\mathrm{e}}}\right)^{2}\right)$,

where $f=n(\mathrm{SiS}) / n\left(\mathrm{H}_{2}\right)$, i.e., the ratio of the number density of $\mathrm{SiS}$ molecules to that of $\mathrm{H}_{2}$ molecules. In CSEs such as these, $\mathrm{H}$ is expected to be mainly in molecular form. In Sect. 4 
Table 3. Model results.

\begin{tabular}{|c|c|c|c|c|c|c|c|c|c|c|c|c|c|c|c|c|}
\hline \multirow[b]{2}{*}{ Source } & \multicolumn{8}{|c|}{ SED modelling } & \multicolumn{4}{|c|}{ CO modelling } & \multicolumn{4}{|c|}{ SiS modelling } \\
\hline & $\begin{array}{c}D \\
{[\mathrm{pc}]}\end{array}$ & $\begin{array}{c}L_{\star} \\
{\left[L_{\odot}\right]}\end{array}$ & $\begin{array}{c}T_{\star} \\
{[\mathrm{K}]}\end{array}$ & $\tau_{10}$ & $\begin{array}{c}T_{\mathrm{c}} \\
{[\mathrm{K}]}\end{array}$ & $\begin{array}{c}r_{\mathrm{i}} \\
{[\mathrm{cm}]}\end{array}$ & $\chi_{\text {red }}^{2}$ & $N$ & $\begin{array}{c}\dot{M} \\
{\left[M_{\odot} \mathrm{yr}^{-1}\right]}\end{array}$ & $\begin{array}{c}v_{\mathrm{e}} \\
{\left[\mathrm{km} \mathrm{s}^{-1}\right]}\end{array}$ & $\chi_{\text {red }}^{2}$ & $N$ & $f_{0}$ & $\begin{array}{l}r_{\mathrm{e}}^{a} \\
{[\mathrm{~cm}]}\end{array}$ & $\chi_{\mathrm{red}}^{2}$ & $N$ \\
\hline \multicolumn{17}{|l|}{ carbon stars } \\
\hline LP And & 630 & 9400 & 2000 & 0.60 & 1100 & $1.8 \times 10^{14}$ & 0.8 & 11 & $1.5 \times 10^{-5}$ & 13.5 & 0.7 & 7 & $1.1 \times 10^{-6}$ & $2.2 \times 10^{16}$ & 2.8 & 3 \\
\hline RV Aqr & 670 & 6800 & 2200 & 0.27 & 1300 & $7.6 \times 10^{13}$ & 0.8 & 9 & $2.8 \times 10^{-6}$ & 15.0 & 0.3 & 3 & $1.5 \times 10^{-6}$ & $9.3 \times 10^{15}$ & $\ldots$ & 1 \\
\hline $\mathrm{U} \mathrm{Cam}^{b}$ & 340 & 7000 & 2700 & 0.01 & 1500 & $4.4 \times 10^{13}$ & 1.5 & 9 & $2.0 \times 10^{-7}$ & 11.5 & $\ldots$ & 4 & $<7.0 \times 10^{-5}$ & $3.0 \times 10^{15}$ & $\ldots$ & 1 \\
\hline S Cep & 380 & 7300 & 2200 & 0.12 & 1400 & $5.8 \times 10^{13}$ & 1.5 & 9 & $1.2 \times 10^{-6}$ & 21.5 & 0.9 & 5 & $<6.0 \times 10^{-6}$ & $4.8 \times 10^{15}$ & $\ldots$ & 1 \\
\hline V Cyg & 310 & 6300 & 1900 & 0.08 & 1200 & $8.7 \times 10^{13}$ & 0.6 & 8 & $9.0 \times 10^{-7}$ & 10.5 & 0.5 & 5 & $3.5 \times 10^{-6}$ & $6.4 \times 10^{15}$ & 0.6 & 2 \\
\hline V821 Her & 600 & 7900 & 2200 & 0.45 & 1500 & $8.1 \times 10^{13}$ & 2.4 & 10 & $1.8 \times 10^{-6}$ & 13.0 & 3.9 & 4 & $5.5 \times 10^{-6}$ & $8.1 \times 10^{15}$ & $\ldots$ & 1 \\
\hline CW Leo & 120 & 9600 & 2000 & 0.90 & 1200 & $1.7 \times 10^{14}$ & 2.1 & 9 & $1.5 \times 10^{-5}$ & 14.0 & 0.5 & 8 & $1.4 \times 10^{-6}$ & $2.2 \times 10^{16}$ & 2.5 & 6 \\
\hline RW LMi & 440 & 9700 & 2000 & 0.50 & 1000 & $2.1 \times 10^{14}$ & 1.4 & 11 & $6.0 \times 10^{-6}$ & 16.5 & 1.1 & 7 & $4.0 \times 10^{-6}$ & $1.3 \times 10^{16}$ & 0.9 & 3 \\
\hline V384 Per & 560 & 8100 & 2000 & 0.25 & 1300 & $1.0 \times 10^{14}$ & 1.5 & 11 & $3.5 \times 10^{-6}$ & 14.5 & 0.7 & 6 & $2.5 \times 10^{-6}$ & $1.1 \times 10^{16}$ & $\ldots$ & 1 \\
\hline R Vol & 730 & 6800 & 2000 & 0.30 & 1500 & $6.6 \times 10^{13}$ & 1.1 & 9 & $1.7 \times 10^{-6}$ & 16.5 & 0.6 & 3 & $<3.0 \times 10^{-5}$ & $7.0 \times 10^{15}$ & $\ldots$ & 1 \\
\hline AFGL 3068 & 980 & 7800 & 2000 & 2.70 & 1100 & $2.5 \times 10^{14}$ & 1.8 & 8 & $1.0 \times 10^{-5}$ & 13.5 & 0.6 & 4 & $3.5 \times 10^{-6}$ & $1.8 \times 10^{16}$ & 6.2 & 3 \\
\hline IRAS 07454-7112 & 710 & 9000 & 2100 & 0.45 & 1200 & $1.4 \times 10^{14}$ & 1.0 & 9 & $5.0 \times 10^{-6}$ & 12.5 & 0.1 & 2 & $6.5 \times 10^{-6}$ & $1.3 \times 10^{16}$ & 2.6 & 2 \\
\hline IRAS 15 & 640 & 9000 & 2200 & 0.80 & 1100 & $1.9 \times 10^{14}$ & 7.0 & 9 & $1.0 \times 10^{-5}$ & 19. & 0.2 & 2 & $3.0 \times 10^{-6}$ & $1.5 \times 10^{16}$ & 7.4 & 3 \\
\hline IRAS 151 & 500 & 8800 & 2400 & 0.55 & 1200 & $1.5 \times 10^{14}$ & 0.4 & 9 & $9.0 \times 10^{-6}$ & 21.0 & 0.9 & 4 & $2.0 \times 10^{-6}$ & $1.4 \times 10^{16}$ & 15.8 & 3 \\
\hline \multicolumn{17}{|l|}{ M-type } \\
\hline TX Cam & 380 & 8600 & 2600 & 1.00 & 1300 & $1.0 \times 10^{14}$ & 1.1 & 8 & $1.0 \times 10^{-5}$ & 18.0 & 1.0 & 5 & $4.0 \times 10^{-7}$ & $1.6 \times 10^{16}$ & 2.0 & 4 \\
\hline R Cas & 110 & 3500 & 2800 & 0.06 & 900 & $1.4 \times 10^{14}$ & 0.3 & 8 & $5.0 \times 10^{-7}$ & & 2.0 & 4 & $4.0 \times 10^{-7}$ & $5.0 \times 10^{15}$ & $\ldots$ & 1 \\
\hline GX Mon & 550 & 8200 & 2600 & 2.00 & 900 & $1.1 \times 10^{14}$ & 5.0 & 9 & $2.0 \times 10^{-5}$ & 18 & 2.0 & 4 & $1.1 \times 10^{-7}$ & $2.2 \times 10^{16}$ & 3.7 & 3 \\
\hline WX Psc & 700 & 10300 & 1800 & 3.00 & 1000 & $1.1 \times 10^{14}$ & 1.3 & 9 & $4.0 \times 10^{-5}$ & 18.5 & 2.0 & 4 & $1.7 \times 10^{-7}$ & $3.0 \times 10^{16}$ & 7.2 & 4 \\
\hline IK Tau & 260 & 7700 & 2400 & 2.00 & 1500 & $7.8 \times 10^{13}$ & 1.0 & 9 & $1.0 \times 10^{-5}$ & 18.0 & 0.7 & 4 & $1.0 \times 10^{-7}$ & $1.6 \times 10^{16}$ & 9.9 & 4 \\
\hline IRC-10529 & 620 & 10600 & 2000 & 3.50 & 1100 & $1.8 \times 10^{14}$ & 2.2 & 8 & $3.0 \times 10^{-5}$ & 13.0 & 4.0 & 5 & $1.0 \times 10^{-7}$ & $3.1 \times 10^{16}$ & 0.1 & 3 \\
\hline IRC +40004 & 680 & 11800 & 2000 & 0.90 & 1100 & $1.5 \times 10^{14}$ & 0.9 & 7 & $1.5 \times 10^{-5}$ & 17.5 & 0.9 & 2 & $3.5 \times 10^{-7}$ & $1.9 \times 10^{16}$ & $\ldots$ & 1 \\
\hline IRC +50137 & 1200 & 9800 & 2000 & 3.50 & 1100 & $1.7 \times 10^{14}$ & 1.2 & 7 & $3.0 \times 10^{-5}$ & 16.5 & 1.0 & 2 & $5.0 \times 10^{-7}$ & $2.0 \times 10^{16}$ & 0.9 & 3 \\
\hline
\end{tabular}

${ }^{a}$ The SiS envelope size is determined from Eq. (3).

${ }^{b} \mathrm{U}$ Cam has a detached shell which complicates the analysis of the present-day mass-loss characteristics (for details see Schöier et al. 2005a).

we discuss deviations from a Gaussian fractional abundance distribution.

The best fit model is found by minimising the total $\chi^{2}$ defined as

$\chi_{\mathrm{tot}}^{2}=\sum_{i=1}^{N}\left[\frac{\left(I_{\mathrm{mod}}-I_{\mathrm{obs}}\right)}{\sigma}\right]^{2}$,

where $I$ is the integrated line intensity and $\sigma$ the uncertainty in the measured value (usually dominated by the calibration uncertainty of $\pm 20 \%$ ), and the summation is done over $N$ independent observations.

The spatial size of the SiS molecular envelopes are assumed to be the same as those for $\mathrm{SiO}$, i.e., these two molecules are assumed to behave in the same way to photodissociation (e.g., Wirsich 1994). González Delgado et al. (2003) and Schöier et al. (2006b) found that a scaling law

$\log r_{\mathrm{e}}=19.2+0.48 \log \left(\frac{\dot{M}}{v_{\mathrm{e}}}\right)$

where $\dot{M}$ is the mass-loss rate and $v_{\mathrm{e}}$ the expansion velocity of the wind, provide a good fit in the case of $\mathrm{SiO}$ for a large sample of M-type and carbon stars. Using this scaling law only one free parameter remains, the fractional abundance of $\operatorname{SiS}\left(f_{0}\right)$. The adopted sizes using Eq. (3) are reported in Table 3. In Sect. 4.3.1 the validity of this approximation is tested for the two carbon stars CW Leo and RW LMi which are the only sources where high angular interferometric observations of SiS line emission exist.

\section{Results}

\subsection{SiS abundances}

The derived abundances for the sample of 19 AGB stars where SiS line emission has been detected are reported in Table 3 and Fig. 2. The fractional abundance of SiS varies substantially between the carbon star (filled circles in Fig. 2) and M-type samples (filled squares in Fig. 2). The mean fractional abundance of $\mathrm{SiS}$ in carbon stars of $3.1 \times 10^{-6}$ (11 objects) is about one order of magnitude higher than in the M-type AGB stars (on average $2.7 \times 10^{-7} ; 8$ objects).

There is a weak trend that the fractional $\mathrm{SiS}$ abundance decreases as the density of the wind $\left(\dot{M} / v_{\mathrm{e}}\right)$ increases. The Pearson correlation coefficients are $r=-0.36$ for the carbon stars and $r=-0.33$ for the M-type AGB stars. In comparison, for the same sample of sources, the derived $\mathrm{SiO}$ abundances have much stronger correlations with $r=-0.60$ for the carbon stars and $r=-0.64$ for the M-type AGB stars. The correlation in the case of $\mathrm{SiO}$ is further increased for the larger sample of sources presented in González Delgado et al. (2003) and Schöier et al. (2006b) and interpreted as increased importance of adsorption of $\mathrm{SiO}$ molecules onto dust grains as the density in the wind increases.

The fractional abundances of SiS derived agrees well, typically within a factor of two, with those obtained by Woods et al. (2003) using a simple excitation model, for the five high massloss rate objects in common. The abundances derived in the present analysis are usually higher than those reported by Woods et al. (2003) possibly a result of their adopted excitation temperature being too high. Bujarrabal et al. (1994) find SiS fractional abundances that on average are higher for the carbon stars 


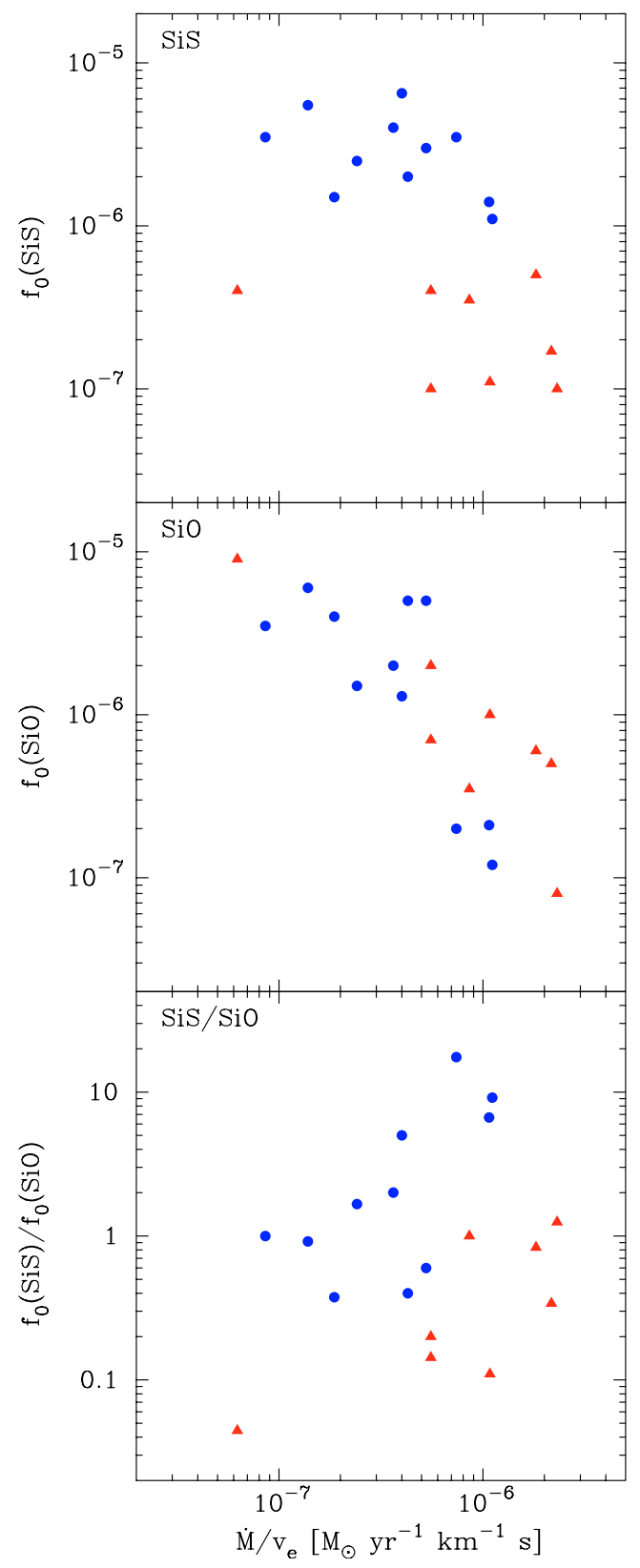

Fig. 2. Upper panel - SiS fractional abundance $\left(f_{0}\right)$ obtained from an excitation analysis, as a function of a density measure $\left(\dot{M} / v_{\mathrm{e}}\right)$, for carbon stars (filled circles) and M-type (O-rich) AGB stars (filled triangles). Middle panel - same as above for $\mathrm{SiO}$ for the same sample of stars (Schöier et al. 2006b; Schöier et al., in prep.). Lower panel - the $\mathrm{SiS} / \mathrm{SiO}$-abundance ratio.

than for the M-type AGB stars, supporting the findings in this paper. However, they only detect $\mathrm{SiS}$ emission for two M-type AGB stars and four carbon stars. The fractional abundances that Bujarrabal et al. (1994) derive, through a simple analysis, agree within a factor of five with the values given in Table 3 for the 6 sources in common.

The fits to the SiS multi-transitional data are generally not as good as for $\mathrm{CO}$ or $\mathrm{SiO}$. As examples we show the best-fit models for the carbon star CW Leo and the M-type AGB star IK Tau in Fig. 3. For CW Leo a reasonably good fit to the observations, both in term of the velocity-integrated line intensity $\left(\chi_{\text {red }}^{2}=2.5\right)$ as well as line profile, is found. In contrast, for IK Tau both the line intensities $\left(\chi_{\text {red }}^{2}=9.9\right)$ and line profiles poorly fit the observations. In particular, for the high- $J$ transitions, where the signal-to-noise is high, the models predict line profiles that are clearly much more flat-topped than what is actually observed. In Sect. 4.3 possible causes for the generally high $\chi^{2}$-values will be discussed, including a compact, high fractional abundance region close to the star which will naturally produce parabolic line profiles.

Results from the SiS excitation analysis in the case of the carbon star CW Leo are shown in Fig. 4, where the excitation temperature, $T_{\mathrm{ex}}$, and the tangential optical depth at the line centre, $\tau_{\tan }$, are plotted as functions of radial distance from the star for the $J=5 \rightarrow 4$ and $J=20 \rightarrow 19$ transitions. It is clear that both lines are formed under non-LTE conditions as $T_{\mathrm{ex}}$ is far from the kinetic temperature of the gas ( $T_{\text {kin }}$, also shown in Fig. 4) where $\tau_{\tan }$ is peaking. Both lines are also optically thin (near the line centre) even in this high mass-loss rate object.

\subsection{Sensitivity analysis}

In the SiS excitation analysis many parameters have been fixed at values obtained from modelling of other molecules (such as $\mathrm{CO}$ and $\mathrm{SiO}$ ) or thermal dust emission. These parameters, such as the mass-loss rate (density structure) and temperature structure have some uncertainties. In Table 4 we report results of an extensive sensitivity analysis where the values adopted for several of the fixed parameters have been varied within reasonable limits, typically $\pm 30 \%$ for the high mass-loss rate carbon star CW Leo and the low mass-loss rate M-type AGB star R Cas. The calculations were performed for a $15 \mathrm{~m}$ telescope (such as the SEST or JCMT) and the velocity-integrated line intensities for selected transitions from each new model were compared to those from the best fit model presented in Table 3.

Varying the mass-loss rate $(\dot{M})$, i.e. the $\mathrm{H}_{2}$ density scale, by $\pm 30 \%$ results in an almost linear response in the line intensities indicating that the emission is more or less optically thin (see also Fig. 4). These changes only marginally affect the line ratios, at most by $10 \%$. A similar result is obtained from varying the fractional SiS abundance $\left(f_{0}\right)$. The line intensities are significantly affected by changes in the SiS envelope size. As expected, it is found that the lower lying (in frequency) $J=5 \rightarrow 4$ and $J=6 \rightarrow 5$ transitions, which probe material at larger radial distances from the star (Fig. 4), are most sensitive to the adopted SiS envelope size. The $J=19 \rightarrow 18$ and $J=20 \rightarrow 19$ line emission are hardly affected. This means that line intensity ratios, and hence the quality of the model in fitting multi-transition observations, will be sensitive to the adopted envelope size, more so than changes in the fractional SiS abundance.

Changing the absolute scale of the kinetic temperature structure, obtained in a self-consistent manner from the $\mathrm{CO}$ analysis, by $\pm 30 \%$ only affects the line intensities by a few percent. This indicates that collisional excitation of the SiS lines is not very effective. This is further supported by variation in the external radiation fields. The excitation analysis includes the possibility of populating also the first vibrationally-excited state of SiS through the absorption of $13 \mu \mathrm{m}$ photons, primarily from dust emission. This can significantly change the derived line intensities of transitions within the ground vibrational state as reported in Table 4. For the high mass-loss rate object CW Leo, the thermal dust emission dominates over the direct stellar radiation field in the excitation. For the low mass-loss rate object R Cas the radiation fields from the star and circumstellar dust appear to be equally important. We also note that the high- $J$ lines are more sensitive to the radiation field than the low- $J$ lines affecting the line intensity ratios. 
F. L. Schöier et al.: SiS in circumstellar envelopes around AGB stars
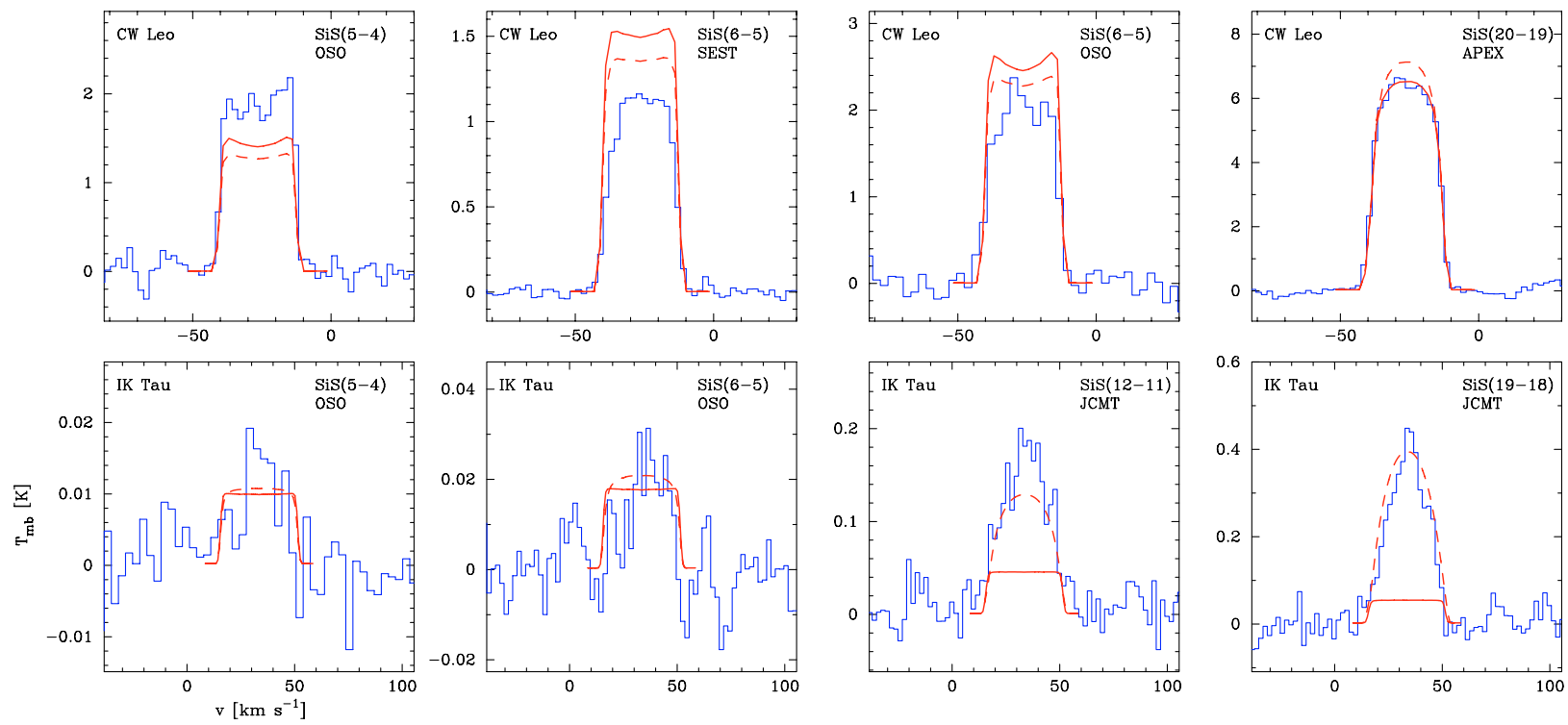

Fig. 3. Best-fit models (solid lines; parameters given in Table 3) for the carbon star CW Leo and the M-type AGB star IK Tau overlayed on observed spectra (histograms). Models with an additional compact, high SiS abundance, pre-condensation component are also shown (dashed line; see text for details).

Table 4. Sensitivity tests for a $15 \mathrm{~m}$ telescope ${ }^{a}$.

\begin{tabular}{|c|c|c|c|c|c|}
\hline Model with & $\begin{array}{c}5 \rightarrow 4 \\
{[\%]}\end{array}$ & $\begin{array}{c}6 \rightarrow 5 \\
{[\%]}\end{array}$ & $\begin{array}{c}12 \rightarrow 11 \\
{[\%]}\end{array}$ & $\begin{array}{c}19 \rightarrow 18 \\
{[\%]}\end{array}$ & $\begin{array}{c}20 \rightarrow 19 \\
{[\%]}\end{array}$ \\
\hline \multicolumn{6}{|l|}{ CW Leo } \\
\hline$\dot{M}+30 \%$ & +28 & +26 & +21 & +22 & +21 \\
\hline$\dot{M}-30 \%$ & -27 & -27 & -26 & -27 & -27 \\
\hline$f_{0}+30 \%$ & +29 & +27 & +21 & +19 & +19 \\
\hline$f_{0}-30 \%$ & -29 & -28 & -25 & -24 & -24 \\
\hline$r_{\mathrm{e}}+30 \%$ & +44 & +35 & +7 & 0 & 0 \\
\hline$r_{\mathrm{e}}-30 \%$ & -42 & -39 & -16 & -5 & -5 \\
\hline$T_{\text {kin }}(r)+30 \%$ & -1 & -1 & +1 & +5 & +5 \\
\hline$T_{\text {kin }}(r)-30 \%$ & +4 & +3 & -3 & -9 & -10 \\
\hline no star & 0 & 0 & -1 & -1 & -1 \\
\hline no dust & +5 & -12 & -37 & -37 & -38 \\
\hline \multicolumn{6}{|l|}{$R$ Cas } \\
\hline$\dot{M}+30 \%$ & +23 & +24 & +31 & +32 & +32 \\
\hline$\dot{M}-30 \%$ & -24 & -25 & -30 & -31 & -31 \\
\hline$f_{0}+30 \%$ & +27 & +27 & +27 & +26 & +25 \\
\hline$f_{0}-30 \%$ & -27 & -28 & -27 & -26 & -26 \\
\hline$r_{\mathrm{e}}+30 \%$ & +45 & +37 & +9 & +2 & +1 \\
\hline$r_{\mathrm{e}}-30 \%$ & -42 & -39 & -16 & -4 & -4 \\
\hline$T_{\text {kin }}(r)+30 \%$ & -4 & -3 & +2 & +7 & +8 \\
\hline$T_{\text {kin }}(r)-30 \%$ & +4 & +3 & -3 & -8 & -9 \\
\hline no star & -6 & -6 & -11 & -15 & -16 \\
\hline no dust & +9 & +1 & -14 & -12 & -12 \\
\hline
\end{tabular}

${ }^{a}$ The percentage change in velocity-integrated intensity relative to the best-fit models that are presented in Table 3.

In an analysis such as this it is very difficult to put absolute errors on the derived fractional abundances of SiS listed in Table 3. However, based on our sensitivity analysis a conservative estimate would be that they are reliable to within a factor of two to three, given that the assumption of a single-Gaussian abundance profile is met. The potential of detecting chemical gradients in the SiS fractional abundance distribution will be further discussed in Sect. 4.3.2.

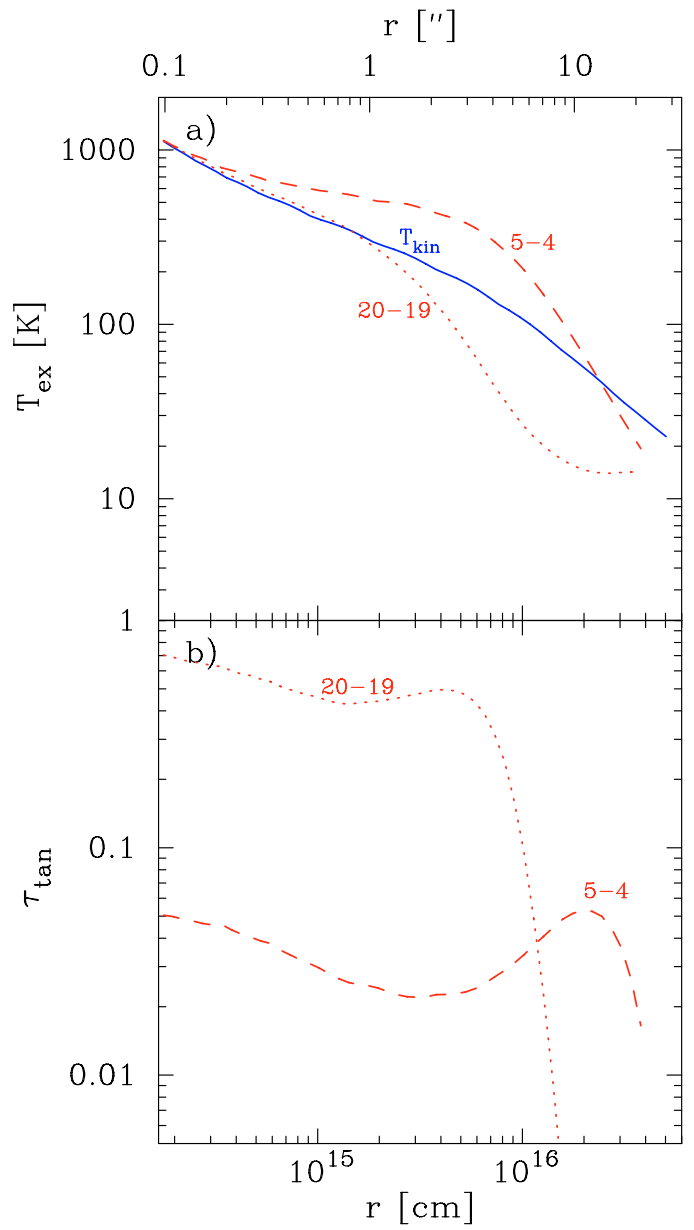

Fig. 4. Results for the best-fit model for CW Leo from the excitation analysis. a) The kinetic temperature of the gas particles as derived from the CO modelling is shown by the solid line. The dotted and dashed lines gives the excitation temperature of the $\operatorname{SiS} J=5 \rightarrow 4$ and $J=20 \rightarrow 19$ transitions, respectively. b) The dotted and dashed lines gives the tangential optical depth $\left(\tau_{\tan }\right)$ at the line centre of the $\mathrm{SiS} J=5 \rightarrow 4$ and $J=20 \rightarrow 19$ transitions, respectively. 


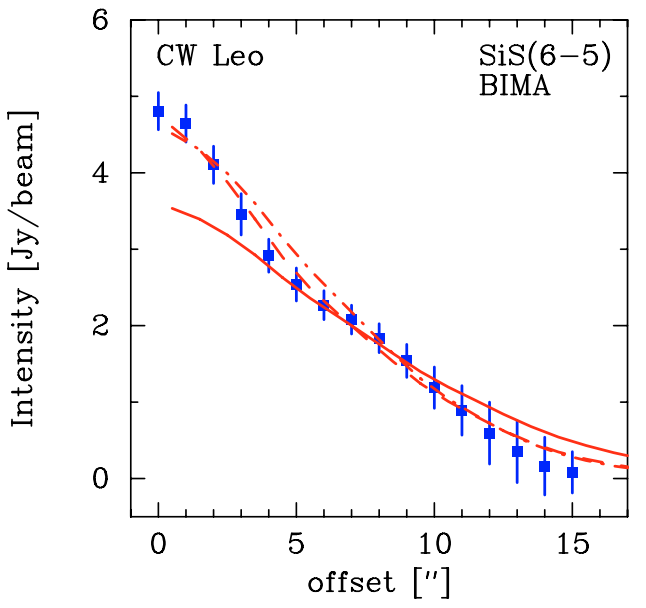

Fig. 5. Azimuthally averaged brightness distribution for CW Leo (IRC+10216), mean value around the systemic velocity from -24 to $-28 \mathrm{~km} \mathrm{~s}^{-1}$ (LSR), obtained using the BIMA interferometer (data from Bieging \& Tafalla 1993) as a function of radial distance to the star in arcsec. The angular resolution is $8^{\prime \prime}(F W H M)$. The best-fit singledish model using $r_{\mathrm{e}}=2.2 \times 10^{16} \mathrm{~cm}$ and $f_{0}=1.5 \times 10^{-6}$ is indicated by the solid line. A model (dash-dotted line) with $r_{\mathrm{e}}=1.7 \times 10^{16} \mathrm{~cm}$ and $f_{0}=2.0 \times 10^{-6}$ better reproduces the interferometer data. A still better fit (dashed line) is obtained by introducing a compact SiS component $\left(r_{\mathrm{c}}=5.0 \times 10^{14} \mathrm{~cm}\right.$ and $\left.f_{\mathrm{c}}=1.7 \times 10^{-5}\right)$ in addition to the more extended envelope with $r_{\mathrm{e}}=1.8 \times 10^{16} \mathrm{~cm}$ and $f_{0}=1.7 \times 10^{-6}$. All models are consistent with available single-dish data within $1 \sigma$.

\subsection{What do the high $\chi_{\text {red }}^{2}$ tell us?}

The resulting $\chi_{\text {red }}^{2}$ are, in the majority of the sources, higher than two, indicating relatively poor fits to the multi-transitional $\mathrm{SiS}$ data. This is in contrast to, e.g., the excitation analysis of $\mathrm{SiO}$ line emission for the same sample of sources, where $\chi_{\text {red }}^{2} \approx 1-2$ in general. There are a number of potential reasons for this that are addressed below.

\subsubsection{SiS envelope size}

In the present analysis we assume that the SiS circumstellar molecular envelope is the same size as that of $\mathrm{SiO}$, i.e., it is assumed that these two molecules respond in the same way to photodissociation. This appears to be a reasonable first approximation (e.g., Wirsich 1994). However, the sensitivity tests performed in Sect. 4.2 show that the line ratios will be affected by the adopted envelope size and might then give poor fits to multitransitional line observations if it is systematically over/underestimated. High angular resolution SiS observations, capable of resolving the emitting regions, exist only for two sources, the carbon stars CW Leo (Bieging \& Tafalla 1993) and RW LMi (Lindqvist et al. 2000). In Figs. 5 and 6 we compare our best fit models from Table 3 with these interferometric observations.

For CW Leo the size estimated from Eq. (3) is $2.2 \times 10^{16} \mathrm{~cm}$, which is clearly too large when compared with the interferometric SiS $J=6 \rightarrow 5$ BIMA observations of Bieging \& Tafalla (1993) as illustrated in Fig. 5 (solid line). If instead the envelope size is lowered by $23 \%$ to $1.7 \times 10^{16} \mathrm{~cm}$ a significantly better fit to the observed brightness distribution is found (Fig. 5; dash-dotted line). When reducing the envelope size the fractional abundance $f_{0}$ was increased by $33 \%$ from $1.5 \times 10^{-6}$ to $2.0 \times 10^{-6}$. This new model also explains the observed multitransitional single-dish data within $1 \sigma$. equivalent spatial scale ["]

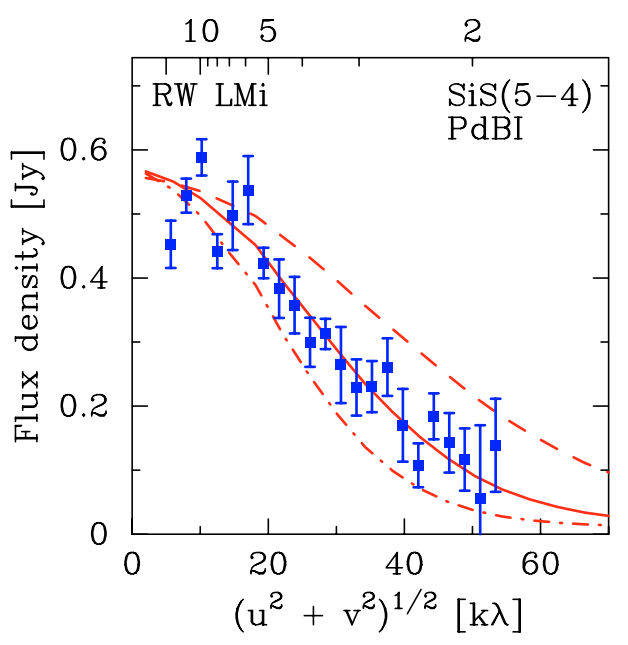

Fig. 6. Azimuthally averaged visibility amplitudes (real part) for RW LMi (CIT6), mean value around the systemic velocity from -2 to $0 \mathrm{~km} \mathrm{~s}^{-1}$ (LSR), obtained using the Plateu de Bure interferometer (data from Lindqvist et al. 2000) as a function of distance to the phase centre in $k \lambda$ (also shown on the upper abscissa is the equivalent spatial resolution in arcseconds). The best-fit single-dish model using $r_{\mathrm{e}}=1.3 \times 10^{16} \mathrm{~cm}$ and $f_{0}=4.5 \times 10^{-6}$ is indicated by the solid line. Also shown are models with a $30 \%$ smaller envelope size $\left(r_{\mathrm{e}}=9.0 \times 10^{15} \mathrm{~cm}\right.$ and $f_{0}=8.5 \times 10^{-6}$; dashed line $)$ and $30 \%$ larger envelope size $\left(r_{\mathrm{e}}=1.7 \times 10^{16} \mathrm{~cm}\right.$ and $f_{0}=2.8 \times 10^{-6}$; dash-dotted line). All models are consistent with available single-dish data within $1 \sigma$.

In Fig. 6 for RW LMi the model is compared to the Plateu de Bure (PdB) interferometric SiS $J=5 \rightarrow 4$ line observations by Lindqvist et al. (2000) in the $(u, v)$-plane in order to maximise both the sensitivity and the angular resolution. The best-fit model with $r_{\mathrm{e}}=1.3 \times 10^{16} \mathrm{~cm}$ (from Eq. (3)) and $f_{0}=4.5 \times 10^{-6}$ (Table 3 ) gives a very good fit to the flux picked up by the PdB interferometer at all baselines (Fig. 6; solid line). Also shown in Fig. 6 are models with a $30 \%$ smaller envelope size $\left(r_{\mathrm{e}}=9.0 \times 10^{15} \mathrm{~cm}\right.$ and $f_{0}=8.5 \times 10^{-6}$; dashed line) and $30 \%$ larger envelope size $\left(r_{\mathrm{e}}=1.7 \times 10^{16} \mathrm{~cm}\right.$ and $f_{0}=2.8 \times 10^{-6}$; dash-dotted line). These models clearly do not reproduce the observations well. All three models are consistent with available single-dish data within $1 \sigma$.

These two examples illustrate the usefulness of angularly resolved emission in further constraining the abundance distribution by lifting the degeneracy between envelope size and fractional abundance which hampers multi-transitional analysis of single-dish data.

Although only two sources have been studied in SiS line emission using interferometers, the fact that both agree within $23 \%$ for the envelope size as estimated by Eq. (3) makes us confident of the adopted approach, and we feel that the overall high $\chi_{\text {red }}^{2}$ found in the SiS multi-transition analysis are generally not due to this adopted value.

\subsubsection{A compact, high-abundance, SiS component?}

From the fact that multi-transitional $\mathrm{SiO}$ excitation analyses show a clear correlation of decreasing circumstellar fractional abundance with the density of the wind $\left(\dot{M} / v_{\mathrm{e}}\right)$ it has been argued that $\mathrm{SiO}$ molecules effectively freeze out onto dust grains in the inner part of the winds from AGB stars (González Delgado et al. 2003; Schöier et al. 2006b). However, it is difficult to place any constraints on the abundance in the pre-condensation 


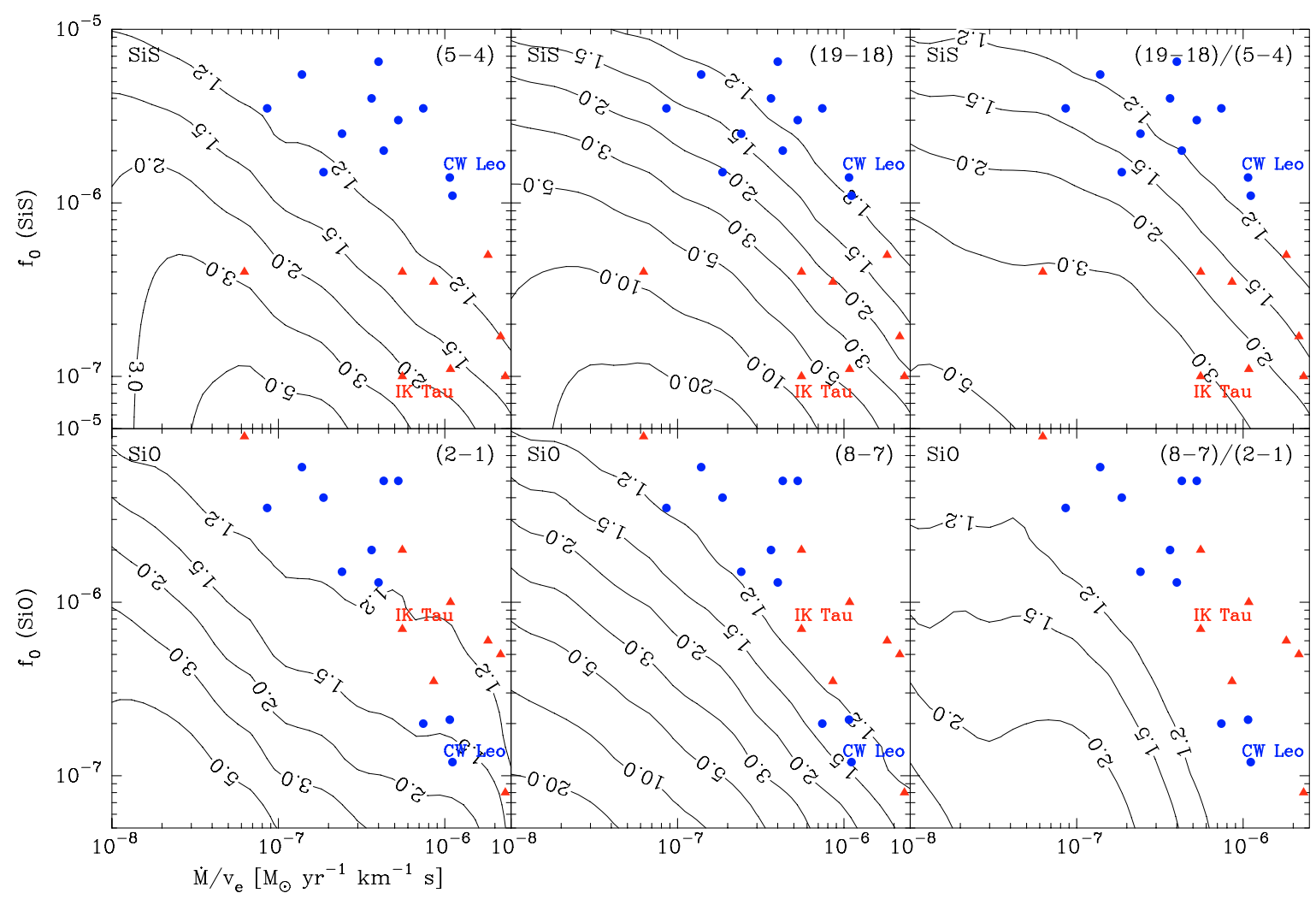

Fig. 7. Sensitivity of $\mathrm{SiS}$ and $\mathrm{SiO}$ circumstellar line emission to the inclusion of an inner compact, high fractional abundance, (pre-condensation) component $\left(f_{\mathrm{c}}=2 \times 10^{-5}\right.$ and $\left.r_{\mathrm{c}}=1 \times 10^{15} \mathrm{~cm}\right)$ as a function of (post-condensation) fractional abundance $\left(f_{0}\right)$ and density of the wind $\left(\dot{M} / v_{\mathrm{e}}\right)$. The contours indicate the ratio of the total line emission for models with and without the compact component (see text for details). Also shown are the fractional abundances $\left(f_{0}\right)$ obtained from the excitation analysis for carbon stars (filled circles) and M-type (O-rich) AGB stars (filled triangles) with the two examples CW Leo and IK Tau high-lighted.

region or its spatial size in the analysis of single-dish data as this only contributes very slightly to the total circumstellar emission (González Delgado et al. 2003). Indeed, high angular resolution interferometric observations are required to further constrain the abundance profiles and solidify the claim of adsorption of $\mathrm{SiO}$ molecules onto dust grains (Schöier et al. 2004; Schöier et al. 2006a).

It is of interest to check whether SiS line emission, as observed by single-dish telescopes in the radio regime, is more sensitive than $\mathrm{SiO}$ to such steep chemical gradients. In Fig. 7 the effect of including a compact region on the line intensity is shown as a function of post-condensation fractional abundance $\left(f_{0}\right)$ and density of the wind $\left(\dot{M} / v_{\mathrm{e}}\right)$. Each panel shows the ratio of line intensities of a model also including a compact, pre-condensation, component (with a constant fractional abundance of $f_{\mathrm{c}}=2 \times 10^{-5}$ out to a cut-off radius $\left.r_{\mathrm{c}}=1 \times 10^{15} \mathrm{~cm}\right)$ to that of a single Gaussian abundance distribution. A grid of models with varying mass-loss rates and fractional abundances in the extended component was then produced. The expansion velocity of the wind was taken to be $12 \mathrm{~km} \mathrm{~s}^{-1}$, a typical value found for AGB stars in general (Ramstedt et al. 2006). Thermal dust grains were also included in the excitation analysis with $\tau_{10}=0.1$. The star was placed at a distance of $300 \mathrm{pc}$ and observations were simulated for $\mathrm{SiO}(J=2 \rightarrow 1$ and $J=8 \rightarrow 7)$ and $\mathrm{SiS}(J=5 \rightarrow 4$ and $J=19 \rightarrow 18$ ) line emission using a $15 \mathrm{~m}$ telescope (such as, the SEST or JCMT). The spatial extents of the SiO and SiS molecular envelopes were calculated using Eq. (3) for each grid point.
In Fig. 7 the fractional abundances of $\mathrm{SiS}$ and $\mathrm{SiO}$ (using a single-Gaussian abundance distribution) found for our sample stars are plotted against the density of the wind $\left(\dot{M} / v_{\mathrm{e}}\right)$. This is a guide to where in the parameter space the objects are found, and how sensitive the observed line emission is expected to be to a steep abundance gradient. From the location of the AGB stars in the panels for $\mathrm{SiO}$ in Fig. 7 (botton row of panels) it is clear that any analysis based on single-dish multi-transitional observations is only weakly sensitive to such a compact component consistent with the findings of González Delgado et al. (2003). While the line intensities may be sensitive by up to $50 \%$ to the inclusion of a compact component, the effect on the $\mathrm{SiO} J=8 \rightarrow 7 / J=2 \rightarrow 1$ line ratio is less than $20 \%$ for all our sample sources. This means that it is generally not possible to separate the compact component from the extended envelope emission from multi-transitional analysis based on single-dish data, and no constraints on the compact component can be found. In addition, the derived post-condensation fractional abundances (using a single-Gaussian abundance profile) may be up to $50 \%$ too high in the worst case (for the majority of sources this effect is less than 20\%).

In contrast, for $\mathrm{SiS}$ it is immediately clear that the results are more sensitive to the inclusion of a compact component (Fig. 7; top row of panels). This is a combination of the lower opacities in the case of the SiS emission and the fact that the transitions readily observed by current instrumentation in the radio regime probe slightly warmer gas closer to the star. For the lines near $345 \mathrm{GHz}$ (Table 1) the SiS $J=19$ energy level is at $166 \mathrm{~K}$, whereas the $\mathrm{SiO} J=8$ level lies at only $75 \mathrm{~K}$. In particular for the M-type AGB stars, where the circumstellar SiS fractional 
abundances are low $\left(\sim 3 \times 10^{-7}\right)$, the inclusion of a compact high abundance component will drastically affect the observed line ratios and hence the quality of the fits to multi-transitional data thereby providing an explanation to the high $\chi_{\text {red }}^{2}$-values reported in Table 3. This sensitivity to chemical gradients also means that while there is the potential to constrain the compact, precondensation, SiS fractional abundance component (if $f_{\mathrm{c}}$ is sufficiently high), the extended, post-condensation, component will have an added uncertainty.

From the location of the M-type star IK Tau in Fig. 7 it is clear that this source may be severely affected by inclusion of a compact, high-abundance component. Indeed, the singleGaussian abundance distribution gives a very poor fit to observations with a reduced $\chi^{2}$ of 9.9 (Table 2 and Fig. 3; solid line). Including a compact component with a constant fractional abundance $f_{\mathrm{c}}$ of $1.1 \times 10^{-5}$ out to a radius $r_{\mathrm{c}}$ of $1.0 \times 10^{15} \mathrm{~cm}$, and at the same time lowering the fractional abundance $f_{0}$ of the extended Gaussian component to $1.0 \times 10^{-8}$, significantly improves the fit to the observations as illustrated in Fig. 3 (dashed line). The reduced $\chi^{2}$ for IK Tau using the two adjustable parameters $f_{\mathrm{c}}$ and $f_{0}$ is 1.8 . It should also be noted that the fit to the various line shapes is significantly improved (better reproducing the parabolic shapes observed), in particular for the high- $J$ transitions where the signal-to-noise is high. This modelling of IK Tau is meant to illustrate the fact that a circumstellar model including the effect of adsorption of SiS molecules onto dust grains can explain the observed line intensities. The actual pre- and post-condensation fractional abundances obtained for IK Tau are uncertain and cannot be uniquely constrained from the limited number of observational constraints available. While this procedure could easily be applied to other sources in our sample, thereby improving the fit to the data, more observational constraints are needed to make this a meaningful approach.

It has been suggested, based on observations of $\mathrm{SiS}$ rovibrational transitions in the infra-red, that the carbon star CW Leo (IRC+10216) has a steep SiS fractional abundance gradient in its inner wind (Boyle et al. 1994). In their modelling, Boyle et al. (1994) find that an initially high fractional abundance of $4.3 \times 10^{-5}$, decreased by an order of magnitude to $4.3 \times 10^{-6}$ at a radial distance of about $8 \times 10^{14} \mathrm{~cm}$ (at an adopted distance of $200 \mathrm{pc}$ ), best reproduces the data. Observations in the radio regime using the $\mathrm{PdB}$ and BIMA interferometers have also been performed (Bieging \& Tafalla 1993; Lucas et al. 1995). Bieging \& Tafalla (1993) find some indications of a compact $\mathrm{SiS}$ component from their interferometric $\mathrm{SiS} J=6 \rightarrow 5$ BIMA observations. In Fig. 5 (dashed line) we show that a model with a compact component with a constant fractional abundance $f_{\mathrm{c}}$ of $1.7 \times 10^{-5}$ out to a radius $r_{\mathrm{c}}$ of $5 \times 10^{14} \mathrm{~cm}$ (corresponding to $8 \times 10^{14} \mathrm{~cm}$ at $200 \mathrm{pc}$ ) provides a somewhat better fit to the BIMA observations than a model without it (dash-dotted line). These values are also fully consistent with those derived by Boyle et al. (1994) when considering their somewhat larger distance and mass-loss rate adopted for CW Leo. The location of CW Leo in Fig. 7 suggests that multi-transitional single-dish observations in the radio regime are not expected to be sensitive enough to any $\mathrm{SiS}$ abundance gradients. This is further illustrated in Fig. 3 where it is not possible to discriminate (the difference is less than the calibration uncertainty of $20 \%$ ) between a model with a compact component used to fit the BIMA data (dotted line) and a model using a single Gaussian abundance distribution (solid line) with parameters reported in Table 2.

We note that Keady \& Ridgway (1993) and Schöier et al. (2006a) found that explaining observed SiO line emission towards CW Leo requires a drastic decrease, by an order of magnitude, in its fractional abundance at a radial distance of about of $5 \times 10^{14} \mathrm{~cm}$ (for a distance of $120 \mathrm{pc}$ ). Recent highfrequency observations of $\mathrm{H}^{13} \mathrm{CN} J=8 \rightarrow 7$ line emission towards CW Leo using the SMA suggest that the fractional abundance of HCN remains more or less constant throughout the inner wind and the extended CSE (Schöier et al. 2007). This lends further support to the interpretation that both $\mathrm{SiO}$ and SiS have large gradients in their fractional abundances and that this is not the effect of drastically varying physical properties, such as density or temperature, in the envelope. Additional observations such as these are required in general for AGB stars in order to obtain more reliable constraints on the SiS abundance distribution.

\subsubsection{Time variability}

It has been shown that SiS line emission can be time variable. Carlström et al. (1990) found that the circumstellar $J=5 \rightarrow 4$ and $J=6 \rightarrow 5$ line emission towards CW Leo varies on a regular basis that follows the light curve in the $K$-band of the star. It was also found that when the $J=5 \rightarrow 4$ line emission was near maximum (also maximum flux in the $K$-band) the $J=6 \rightarrow 5$ line emission was near its minimum. The variation in line intensity between maximum and minimum is about a factor of two. Thus observations of these lines at different epochs, which is usually the case in the present analysis, will significantly degrade the quality of the fit of the model to the observations. A plausible explanation for the time variability of certain SiS transitions is overlap of vibrational bands in the infra-red with another molecular species. This will have the effect that the vibrationally excited levels of SiS will be pumped by an additional, but selective, source modifying the excitation in the ground vibrational state. Recent observations of possible maser emission in the SiS $J=11 \rightarrow 10, J=14 \rightarrow 13, J=15 \rightarrow 14$ lines by Fonfría Expósito et al. (2006) towards CW Leo identify existing overlap of ro-vibrational transitions in the mid-infrared of $\mathrm{SiS}$ with other abundant molecules such as $\mathrm{C}_{2} \mathrm{H}_{2}$ and $\mathrm{HCN}$ as a realistic pumping mechanism.

We find no direct evidence of any maser action in the SiS $J=20 \rightarrow 19$ line emission observed towards CW Leo using the APEX telescope. This could mean that this transition is not affected by the selective excitation due to the overlaps. Alternatively it could mean that the relatively large beam of the APEX telescope, compared with the IRAM $30 \mathrm{~m}$ telescope, dilutes the emission from the region very close to the star where the masers are thought to be active. Fonfría Expósito et al. (2006) derive expansion velocities of about $10 \mathrm{~km} \mathrm{~s}^{-1}$ for their observed lines indicating that they are mainly produced in the acceleration zone $\left(\approx 5-7\right.$ stellar radii) before the terminal velocity $\left(14 \mathrm{~km} \mathrm{~s}^{-1}\right)$ of the wind is reached. From modelling of our transitions it is clear that we are not directly probing this region of the wind (see Fig. 3). Currently, the effect of time variability due to maser emission is unknown for AGB stars in general.

\section{Circumstellar chemistry}

Stellar atmosphere models predict that the SiS fractional abundance in AGB stars is strongly dependent on the photospheric C/O-ratio (e.g., Millar 2003; Cherchneff 2006). Typically, SiS fractional abundances under the condition of LTE carbon are expected to be $\sim 10^{-5}$ in carbon stars whereas in M-type AGB-stars they are expected to be only $\sim 10^{-8}-10^{-7}$. From the results reported in Table 3 it is clear that the citcumstellar fractional abundance of SiS varies substantially between the carbon 
star and M-type samples. The mean fractional abundances of $\mathrm{SiS}$ found in the present study are in reasonable agreement with LTE stellar atmosphere chemistries. For carbon stars the mean is $3.1 \times 10^{-6}$, about one order of magnitude higher than that of the M-type AGB stars (on average $2.7 \times 10^{-7}$ ).

Based on the present modelling, using a single Gaussian abundance profile, there is a weak trend that the $\mathrm{SiS}$ fractional abundance for the sample sources decreases as $\dot{M} / v_{\mathrm{e}}$, i.e. the density, in the wind increases (Fig. 2; top panel). A much stronger correlation is found for the related molecule SiO (González Delgado et al. 2003; Schöier et al. 2006b) as shown in Fig. 2 (middle panel) for the stars also detected in $\mathrm{SiS}$. This has been interpreted as effective adsorption of $\mathrm{SiO}$ molecules onto dust grains in the inner wind which has been further corroborated by interferometric observations (Schöier et al. 2004; Schöier et al. 2006a). In addition, there appears to be no way of distinguishing a C-rich chemistry from that of an O-rich chemistry based on an estimate of the circumstellar $\mathrm{SiO}$ abundance alone, suggesting that non-equilibrium chemical processes are important. For SiS the direct interpretation of the derived circumstellar fractional abundances is more complicated as discussed in Sect. 4.3, which could explain the weaker correlation of its fractional abundance with the density of the wind.

A departure from LTE is to be expected by the variable nature of AGB stars which induces shock waves that propagate through the photosphere thereby affecting its chemistry. Models of shocked stellar atmospheres (Willacy \& Cherchneff 1998; Duari et al. 1999; Cherchneff 2006) indicate that the fractional abundances of molecules, such as $\mathrm{SiO}$ and $\mathrm{SiS}$, can be significantly altered by the passage of periodic shocks. Willacy \& Cherchneff (1998) in their chemical modelling of CW Leo $($ IRC +10216$)$ find a thermal equilibrium value for the SiS fractional abundance (relative to $\mathrm{H}_{2}$ ) of $1.5 \times 10^{-5}$ which is increased to $3.4 \times 10^{-5}$ due to non-equilibrium chemical processes. For comparison, Millar (2003) obtains an LTE SiS fractional abundance of $1.0 \times 10^{-5}$. In the present analysis of multi-transitional SiS line emission towards CW Leo we derive a lower circumstellar value of $1.4 \times 10^{-6}$, using a single Gaussian abundance component (Sect. 4 and Table 3). However, as discussed in Sect. 4.3.2 there is observational evidence that the fractional abundance closer to the star may be as high as $1.7 \times 10^{-5}$ (Fig. 5) and is more in line with the chemical model predictions. The low SiS fractional abundance in the extended, postcondensation, part of the envelope indicates that SiS molecules, such as $\mathrm{SiO}$ molecules, are significantly incorporated into dust grains.

Similarly, Duari et al. (1999) found a fractional SiS abundance (relative to $\mathrm{H}_{2}$ ) equilibrium value of $4.4 \times 10^{-7}$ in their chemical modelling of the M-type AGB star IK Tau which is initially increased by an order of magnitude to $4.8 \times 10^{-6}$ near the photosphere due to shocks but is significantly reduced to $8.8 \times 10^{-10}$ before the dust formation zone. In comparison, Cherchneff (2006) derived a significantly lower LTE value of $7.1 \times 10^{-9}$ for the same C/O-ratio of 0.75 and a non-equilibrium value near the photosphere of $1.8 \times 10^{-6}$. Also, a drastic decrease of the SiS fractional abundance in the chemically and dynamically active region between $1-5$ stellar radii, before dust formation sets in, is found by Cherchneff (2006) who derive a very low fractional abundance of $\approx 1.0 \times 10^{-8}$ for their synthetic model of TX Cam. In our excitation analysis we derive circumstellar SiS fractional abundances of $1.0 \times 10^{-7}$ for IK Tau and $4.0 \times 10^{-7}$ for TX Cam, however, the fits to multi-transitional SiS data are poor, in particular for IK Tau. A significantly better fit is obtained if the fractional abundance close to the star is drastically increased, in the case of IK Tau up to $1.1 \times 10^{-5}$ assuming a size of this component of $1.0 \times 10^{15} \mathrm{~cm}$ (Sect. 4.3 .2 and Fig. 3). Such a high fractional abundance in the inner wind would favour the importance of non-equilibrium processes. Presently, it is not clear if the very low fractional abundance $\left(1.0 \times 10^{-8}\right)$ found in the extended envelope around IK Tau is a result of non-equilibrium chemistry in the inner wind or a result of freeze-out onto dust grains. Interferometric observations are clearly required to make further progress and better constrain the chemical models. Also the estimated chemical reaction rates involving Si might have to be re-examined (Cherchneff 2006).

\section{Conclusions}

A systematic and extensive excitation analysis of multitransition (sub-) millimetre SiS line emission was performed for a large sample of 19 AGB stars with a range of photospheric $\mathrm{C} / \mathrm{O}$-ratios and mass-loss rates. By constraining the circumstellar SiS abundance distribution we reach the following conclusions:

- In order to derive reliable fractional abundances of SiS, excitation through IR ro-vibrational transitions, needs to be taken into account. This will in most cases (for our sample sources) mean that the effect of dust grains needs to be included in the excitation analysis. Our study presents a thorough treatment of this.

- The adopted SiS envelope size will also affect the quality of the abundance estimates and was tested against available interferometric $\mathrm{SiS}$ line observations for the two carbon stars CW Leo (IRC+10216) and RW LMi (CIT6). We found that the empirical formula derived from $\mathrm{SiO}$ observations is also adequate to use for $\mathrm{SiS}$, i.e., these two molecules appear to respond similarly to photodissociation.

- The mean fractional abundance of SiS in carbon stars of $3.1 \times 10^{-6}$ is about an order of magnitude higher than that found for the M-type AGB stars (on average $2.7 \times 10^{-7}$ ). Taken at face value, these circumstellar fractional abundances are in reasonable agreement with photospheric LTE chemical models. However, there are indications that the SiS fractional abundance could be significantly higher closer to the star which, at least in the case of M-type AGB stars, would require non-equilibrium chemical processes.

- No clear trend that the SiS fractional abundance decreases as the density $\left(\dot{M} / v_{\mathrm{e}}\right)$ of the wind increases is found, in contrast to the results for $\mathrm{SiO}$ for the same sample of stars. This could mean that SiS molecules are less likely to adsorb onto dust grains than $\mathrm{SiO}$ molecules. However, possible time-variability of the line emission in the lower $J$ transitions and the sensitivity of the line emission to abundance gradients in the inner part of the CSE may mask a correlation with the density of the wind. Evidence supporting the presence of steep circumstellar abundance gradients is presented for the carbon star CW Leo and the M-type AGB star IK Tau, which can naturally be explained by effective freezeout of SiS molecules onto dust grains at distances of $\sim 5$ stellar radii.

While it is clear that SiS line emission is a useful tool in investigating the conditions occuring in the chemically and dynamically active region, where dust grains are formed and the stellar wind is accelerated, many uncertainties remain. In particular, the constraints on the detailed SiS abundance distribution are poor. To make significant progress, interferometric observations of both low- and high- $J \mathrm{SiS}$ line emission in order to more firmly 
establish the relative importance of freeze-out onto dust grains, photodissociation, and circumstellar chemistry are required. We note that for a typical stellar distance of $300 \mathrm{pc}$, next generation interferometers such as ALMA will be required to fully resolve the region over which $\mathrm{SiS}$ could effectively freeze-out (angular size of $\left.\approx 0.2-0.4^{\prime \prime}\right)$.

Acknowledgements. The authors are grateful to the staff at the APEX telescope. The referee, J.H. Bieging, is thanked for insightful comments which helped improve the paper. FLS and HO acknowledge financial support from the Swedish Research Council.

\section{References}

Agúndez, M., \& Cernicharo, J. 2006, ApJ, 650, 374

Bieging, J. H., \& Tafalla, M. 1993, AJ, 105, 576

Bieging, J. H., Shaked, S., \& Gensheimer, P. D. 2000, ApJ, 543, 897

Bohren, C. F., \& Huffman, D. R. 1983, Absorption and scattering of light by small particles (New York: Wiley)

Boyle, R. J., Keady, J. J., Jennings, D. E., Hirsch, K. L., \& Wiedemann, G. R. 1994, ApJ, 420, 863

Bujarrabal, V., Fuente, A., \& Omont, A. 1994, A\&A, 285, 247

Carlström, U., Olofsson, H., Johansson, L. E. B., Nguyen-Q-Rieu, \& Sahai, R. 1990, in From Miras to Planetary Nebulae: Which Path for Stellar Evolution?, ed. M. O. Mennessier, \& A. Omont, 170

Cherchneff, I. 2006, A\&A, 456, 1001

Duari, D., Cherchneff, I., \& Willacy, K. 1999, A\&A, 341, L47

Fonfría Expósito, J. P., Agúndez, M., Tercero, B., Pardo, J. R., \& Cernicharo, J. 2006, ApJ, 646, L127

Ford, K. E. S., Neufeld, D. A., Schilke, P., \& Melnick, G. J. 2004, ApJ, 614, 990

Glassgold, A. E. 1999, in Asymptotic Giant Branch Stars, IAU Symp., 191, 337

González Delgado, D., Olofsson, H., Kerschbaum, F., et al. 2003, A\&A, 411, 123

Groenewegen, M. A. T., de Jong, T., \& Baas, F. 1993, A\&AS, 101, 513

Groenewegen, M. A. T., \& Whitelock, P. A. 1996, MNRAS, 281, 1347

Hasegawa, T., Kwok, S., Koning, N., et al. 2006, ApJ, 637, 791

Höfner, S., \& Andersen, A. C. 2007, A\&A, 465, L39

Ivezić, Ž., \& Elitzur, M. 1997, MNRAS, 287, 799

Keady, J. J., \& Ridgway, S. T. 1993, ApJ, 406, 199
Kerschbaum, F. 1999, A\&A, 351, 627

Lindqvist, M., Olofsson, H., Winnberg, A., \& Nyman, L. Å. 1992, A\&A, 263, 183

Lindqvist, M., Schöier, F. L., Lucas, R., \& Olofsson, H. 2000, A\&A, 361, 1036

Lucas, R., Guelin, M., Kahane, C., Audinos, P., \& Cernicharo, J. 1995, Ap\&SS, 224, 293

Marvel, K. B. 2005, AJ, 130, 261

Melnick, G. J., Neufeld, D. A., Ford, K. E. S., Hollenbach, D. J., \& Ashby, M. L. N. 2001, Nature, 412, 160

Millar, J. 2003, in Asymptotic giant branch stars, ed. H. J. Habing, \& H. Olofsson, Astronomy and astrophysics library (New York, Berlin: Springer), 247

Olofsson, H., Lindqvist, M., Nyman, L.-Å., \& Winnberg, A. 1998, A\&A, 329, 1059

Olofsson, H., González Delgado, D., Kerschbaum, F., \& Schöier, F. L. 2002, A\&A, 391, 1053

Ramstedt, S., Schöier, F. L., Olofsson, H., \& Lundgren, A. A. 2006, A\&A, 454, L103

Scalo, J. M., \& Slavsky, D. B. 1980, ApJ, 239, L73

Schöier, F. L., \& Olofsson, H. 2001, A\&A, 368, 969

Schöier, F. L., Ryde, N., \& Olofsson, H. 2002, A\&A, 391, 577

Schöier, F. L., Olofsson, H., Wong, T., Lindqvist, M., \& Kerschbaum, F. 2004, A\&A, 422, 651

Schöier, F. L., Lindqvist, M., \& Olofsson, H. 2005a, A\&A, 436, 633

Schöier, F. L., van der Tak, F. F. S., van Dishoeck, E. F., \& Black, J. H. 2005b, A\&A, 432, 369

Schöier, F. L., Fong, D., Olofsson, H., Zhang, Q., \& Patel, N. 2006a, ApJ, 649, 965

Schöier, F. L., Olofsson, H., \& Lundgren, A. A. 2006b, A\&A, 454, 247

Schöier, F. L., Fong, D., Bieging, J., et al. 2007, ApJ, accepted [arXiv:0707.4364]

Suh, K. 2000, MNRAS, 315, 740

Tenenbaum, E. D., Apponi, A. J., Ziurys, L. M., et al. 2006, ApJ, 649, L17

van Zadelhoff, G.-J., Dullemond, C. P., van der Tak, F. F. S., et al. 2002, A\&A, 395,373

Willacy, K. 2004, ApJ, 600, L87

Willacy, K., \& Cherchneff, I. 1998, A\&A, 330, 676

Wirsich, J. 1994, ApJ, 424, 370

Woitke, P. 2006, A\&A, 460, L9

Woods, P. M., Schöier, F. L., Nyman, L.-Å., \& Olofsson, H. 2003, A\&A, 402, 617 